\title{
Enseignement des sciences, interdisciplinarité et territoire apprenant : Une recherche-action sur le territoire insulaire corse
}

Laure Moretti

\section{(2) OpenEdition}

Journals

Édition électronique

URL : https://journals.openedition.org/ere/8428

DOI : 10.4000/ere.8428

ISSN : 2561-2271

Éditeur

Centr'ERE

\section{Référence électronique}

Laure Moretti, « Enseignement des sciences, interdisciplinarité et territoire apprenant : Une rechercheaction sur le territoire insulaire corse », Éducation relative à l'environnement [En ligne], Volume 16-2 I 2021, mis en ligne le 14 octobre 2021, consulté le 11 février 2022. URL : http:// journals.openedition.org/ere/8428; DOI : https://doi.org/10.4000/ere.8428

Ce document a été généré automatiquement le 11 février 2022.

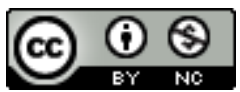

La revue Éducation relative à l'environnement est mise à disposition selon les termes de la Licence Creative Commons Attribution - Pas d'Utilisation Commerciale 4.0 International. 


\title{
Enseignement des sciences, interdisciplinarité et territoire apprenant : Une recherche-action sur le territoire insulaire corse
}

\author{
Laure Moretti
}

1 Tout a commencé par des observations menées en tant qu'enseignante via la stratégie de l'observation participante (Lapassade, 2002). Lors de sorties pédagogiques, nous avons constaté une distance des élèves au territoire et une carence de comportements écocitoyens. Or, la distance au territoire peut-elle expliquer la négligence écocitoyenne? Cette question a été à l'origine d'une recherche-action (Allard-Poesi et Perret, 2003), menée dans le cadre d'une recherche doctorale ${ }^{1}$ et poursuivant deux objectifs : 1) la modélisation et la mise en œuvre d'une stratégie éducative visant une écocitoyenneté plus responsable et engagée; 2) l'évaluation de ses impacts sur les élèves. Les questions spécifiques suivantes ont été formulées: une proposition d'évolution de la forme scolaire actuelle, utilisant l'enseignement des sciences comme point d'ancrage et d'armature pour le développement d'un territoire apprenant initié depuis l'École, aboutirait-elle à une valorisation des liens patrimoniaux ? Ces nouveaux liens au territoire seraient-ils associés à des profils d'écocitoyenneté différents? Cet article se concentre sur les impacts de la stratégie éducative expérimentée sur les liens patrimoniaux. La première partie présente l'action menée, la seconde explicite la méthodologie de recherche et la troisième expose les résultats ainsi que des éléments de réponses aux questions posées. 


\section{La modélisation et la mise en œuvre d'une stratégie éducative basée sur la construction d'un territoire apprenant}

2 Cette section est subdivisée en trois sous-parties. La première présente la démarche de modélisation de la stratégie à partir de l'analyse du contexte d'étude permettant de préciser ses objectifs et ses caractéristiques. La deuxième sous-partie expose les modalités de mise en œuvre et la troisième propose une analyse de la stratégie modélisée au regard de la forme scolaire.

\section{L'analyse du contexte d'étude}

3 La première analyse $\mathrm{du}$ contexte a consisté en une étude sociohistorique des particularités du territoire insulaire corse, axée sur les thématiques des liens patrimoniaux, du sentiment d'appartenance et des comportements écocitoyens. Les comportements écocitoyens engagés des Corses sont expliqués par une îléité corse singulière, caractérisée par de forts liens au territoire, considéré comme un système socio-patrimonial (Sébastien, 2014). Nous avons alors émis l'hypothèse qu'une stratégie éducative pourrait intervenir en complément de l'éducation à la citoyenneté scolaire actuelle et cibler la valorisation des liens patrimoniaux afin de contribuer à développer une écocitoyenneté engagée. Ainsi, notre action prendrait la forme d'une stratégie éducative considérant le patrimoine comme un levier de développement (Verdoni, 2013) pour la formation d'écocitoyens, conscients et compétents sur leur territoire, puis par extension sur d'autres.

4 Afin d'envisager l'élaboration d'une telle stratégie éducative, nous avons effectué une seconde analyse, axée sur l'étude du système éducatif français actuel. Nous y avons souligné trois conséquences majeures de la forme scolaire (Vincent, Lahire, et Thin, 1994) : la vision de l'éducation scolaro-centrée, la distance École-territoire née du postulat d'un territoire-entrave au projet d'émancipation (Bier 2010; Barthes 2020) et le mode scolaire de socialisation. Ces conséquences sont à nuancer, car les travaux recensés sur la forme scolaire ne tiennent pas ou peu compte des grandes réformes éducatives des années 80/90, engageant le système éducatif dans la voie de la décentralisation éducative et $\mathrm{y}$ développant principalement les stratégies de coéducation et de territorialisation éducative. À ce titre, certains auteurs comme Maryvonne Dussaux considèrent que les réformes visant la coéducation, s'attaquant à la vision de l'éducation scolaro-centrée, ont porté leurs fruits. Selon cette dernière, l'École n'a plus le monopole de l'éducation, aujourd'hui en responsabilité partagée entre différents acteurs éducatifs: elle propose de remplacer le terme de forme scolaire, désormais obsolète, par le terme de « forme éducative » (Dussaux, 2011, p. 12). A contrario, les stratégies de territorialisation éducative, s'attaquant à la distance Écoleterritoire, sont aujourd'hui encore largement critiquées. Pour Michel Develay, la distance École-société (composante sociale du territoire) est en partie à l'origine d'une perte de sens de l'École (Develay, 2007, p. 8). Selon Catherine Becchetti-Bizot (2018), les stratégies de territorialisation éducative n'ont conduit qu'à des érosions éparses de la frontière entre ces deux territoires et méritent des efforts supplémentaires. Enfin, le mode scolaire de socialisation est également critiqué, notamment par Guy Vincent (2008) qui le considère incompatible avec une socialisation démocratique, nécessaire à la 
formation d'un citoyen compétent en démocratie. L'École est donc encore trop distante de son territoire et ne génère pas un mode de socialisation propice à la formation d'un citoyen compétent.

5 Cette seconde analyse nous conduit à confirmer les objectifs de notre action et à en préciser les modalités. Cette action ambitionne la modélisation et la mise en œuvre d'une stratégie éducative visant une écocitoyenneté plus responsable et engagée. Un cahier des charges est fixé : la stratégie éducative vise à contrer les trois conséquences précitées de la forme scolaire actuelle par la combinaison des principes de coéducation, de territorialisation éducative, ainsi que par la valorisation d'un mode de socialisation démocratique. Ce dernier est fondé sur deux piliers majeurs, soit la discussion et l'indétermination (Vincent, 2008), autrement dit, sur un raisonnement rationnel et ouvert, indispensable à une écocitoyenneté de qualité. Nous avons émis l'hypothèse qu'une stratégie éducative, basée sur la construction d'un territoire apprenant (TA) initié depuis l'École, fondé sur une éducation relative à l'environnement valorisant les patrimoines culturels et naturels locaux, permettrait de répondre aux trois exigences du cahier des charges.

6 Selon Jean-Pierre Jambes, l'échelon local est un outil du développement territorial pour créer des plus-values sur, par et pour le territoire. Le TA est alors « une modalité particulière d'organisation du territoire pour augmenter sa capacité à apprendre et à faire.» (Jambes, 2001). Cette définition du TA pourrait induire la vision d'une stratégie éducative au service du développement territorial, de sa valorisation économique et/ou de son aménagement. Face à cette « dimension utilitariste de l'éducation au service des territoires» (Barthes, Maximin et Alpe 2015, p. 9), source de «l'effacement total ou partiel du projet éducatif devant l'action de valorisation territoriale » (Ibid, p. 9), nous souhaitons préciser les finalités de la stratégie éducative ainsi que les moyens développés pour se préserver de cette dérive utilitariste.

7 Sa finalité première est bien éducative: elle vise une valorisation des liens patrimoniaux des élèves et de manière plus globale, le développement d'une écocitoyenneté plus responsable et engagée. Cette finalité éducative n'est cependant pas incompatible avec une finalité relative au développement territorial, le territoire pouvant bénéficier en retour de comportements éco-responsables et engagés de ses acteurs. Par ailleurs, les acteurs hors-École pouvant être à l'origine de cette dérive utilitariste de l'éducation, d'une "éducation "pour" le patrimoine» (Ibid, p. 4), la première tactique d'évitement a consisté en l'établissement d'un TA dont les projets sont initiés et cadrés depuis l'École, les enseignants veillant à recentrer la stratégie éducative sur une éducation sur et par le patrimoine (Ibid), sur des finalités premières éducatives, notamment grâce à l'établissement de "balises curriculaires " (Barthes, Blanc-Maximin et Dorier 2019, p. 3). Ces dernières permettent d'expliciter les visées éducatives, soit dans notre cas, le développement d'une réflexion critique, rationnelle et ouverte.

Dans ce travail, nous considérons un TA comme une organisation apprenante à l'échelle $\mathrm{du}$ territoire. Pour cette dernière, les propositions de définitions sont multiples (Garvin, 1993 ; SOL France, 2015 ; Senge, 2015 ; Becchetti-Bizot et coll., 2017). Dans l'acception choisie, elle se définit comme une organisation humaine capable d'apprendre, c'est-à-dire d'acquérir des savoirs, voire de les créer, puis de les transmettre à l'ensemble de ses membres. C'est aussi une organisation capable de les mettre en pratique sous la forme d'actions permettant une meilleure adaptation au 
territoire. Les membres y sont en interaction et forment un collectif : ils collaborent et coopèrent, développant l'intelligence individuelle comme collective. Il s'agit donc d'une organisation priorisant la justification axiologique de l'apprentissage sur la justification instrumentale, autrement dit le développement de savoirs et valeurs sur les comportements visés (Forquin 2008). La communication horizontale est favorisée et prend le pas sur la communication verticale, chaque membre pouvant apprendre d'un autre, sans distinction hiérarchique. Plus précisément, l'apprentissage par un membre facilite l'apprentissage d'un autre, car le premier aura préalablement documenté et partagé son savoir.

Dans cette recherche, le TA initié par une équipe d'acteurs de l'établissement scolaire, se fonde sur une valorisation de l'éducation à l'environnement proche, ou territoire de proximité, via l'étude, la création et le partage de ressources sur le patrimoine naturel et culturel local. Selon le système de transmission curriculaire (Barthes, BlancMaximin, et Dorier 2019), le curriculum prescrit porte ici sur les éléments patrimoniaux locaux et le développement des capacités de raisonnement rationnel et ouvert ainsi que la capacité de discussion. Le curriculum produit évalué dans cet article s'intéresse à l'impact sur les liens patrimoniaux des élèves. Le curriculum caché ou "signifiant politique non exprimé d'un curriculum» (Ibid, p. 10) porte sur la transformation du profil écocitoyen des élèves. Ce dernier n'est pas présenté dans cet article, mais a été étudié afin d'opérer une vérification des impacts de l'action dans le champ éducatif.

\section{Les piliers fondateurs du territoire apprenant modélisé}

Trois piliers servent d'assises à la modélisation de la stratégie de territoire apprenant.

\section{Pilier 1 : Un ancrage dans l'enseignement des sciences}

10 Nous considérons que l'enseignement des sciences est doté de deux propriétés, aujourd'hui sous-exploitées dans l'enseignement traditionnel, constituant deux qualités qui répondent aux exigences fixées dans le cahier des charges de l'action. Dans un premier temps, nous considérons cet enseignement propice au développement d'une socialisation démocratique. En effet, il impose une démarche d'investigation rigoureuse aux élèves. Ils prennent alors conscience qu'une connaissance n'est fiable qu'une fois issue d'une démonstration faisant usage de la raison et qu'une fois soumise à un examen critique d'experts, discutant de sa validité. Enseigner les sciences, c'est permettre l'usage et l'apprentissage de la raison, car «la science, c'est la raison humaine en action » (Durkheim, 2014, p. 389). Les élèves apprennent également qu'une connaissance scientifique n'est fiable que jusqu'à ce que le contraire soit démontré. Cette vision est notamment soutenue par les contenus d'histoire des sciences des programmes officiels, permettant l'apprentissage de l'ouverture d'esprit. Ainsi, l'enseignement des sciences se base en réalité sur les deux fondements de la socialisation démocratique : la discussion et l'indétermination. L'indétermination ouvre la réflexion de tous au débat et la discussion apporte l'argumentation rationnelle, les deux étant nécessaires au fondement de l'agora démocratique.

11 En ce sens, nous considérons que l'enseignement des sciences permettrait d'initier une conversion vers le mode de socialisation démocratique, offrant la possibilité aux élèves de développer un raisonnement rationnel et ouvert, indispensable à l'écocitoyen. Plus précisément, ce n'est pas le contenu scientifique en lui-même mais la méthode 
scientifique qui permet le développement de cette capacité : selon Durkheim, elle est d'ailleurs « un inestimable instrument de culture logique» (Durkheim, 2014, p. 391). Dans un second temps, nous considérons l'enseignement des sciences propice à l'ancrage territorial, car son contenu, qu'il soit biologique ou géologique, physique ou chimique, est directement corrélable au territoire, basé sur l'étude de l'un de ses éléments concrets. Nous avons donc émis le postulat que le $T A$, exigeant un ancrage territorial, peut y être enraciné par l'enseignement des sciences. Ainsi, l'enseignement des sciences devient un des piliers articulant le $T A$ à la forme scolaire par ses qualités lui permettant d'établir une éducation ancrée territorialement et développant une socialisation démocratique, exigées dans le cahier des charges. Le TA constitue alors la démarche complémentaire par laquelle l'enseignement des sciences développe son plein potentiel. L'enseignement des sciences constituerait le porte-greffe, doté de qualités mais peu productif; le TA constituerait le greffon, offrant une production de qualité supérieure.

\section{Pilier 2 : L'extension obligatoire vers l'interdisciplinarité}

12 Nous considérons l'extension vers l'interdisciplinarité indispensable à l'exigence de coéducation. En effet, le TA impose aux élèves un travail d'équipe. Le thème de recherche sur le patrimoine local, choisi par les élèves, fait l'objet de multiples réflexions, différant les unes des autres selon les sensibilités, représentations, intérêts, valeurs et expériences de chacun. Ces réflexions offrent alors une diversité et donc une richesse de points de vue qui, pour être considérés, appellent une vision large, interdisciplinaire. De plus, le TA exige également une prise de contact avec les acteurs du territoire, les institutions ou les citoyens hors-École considérés comme les experts locaux du thème de recherche. Or, les savoirs ne sont morcelés et cloisonnés en disciplines qu'au sein de l'École et les experts ont une vision beaucoup plus globale, interdisciplinaire. Ainsi, afin d'optimiser les communications interacteurs, le fonctionnement en interdisciplinarité nous a paru une condition du bon fonctionnement du TA. Par ailleurs, l'ancrage du thème choisi sur le territoire et donc sur le réel, nécessite une vision capable d'analyser un système complexe, appelant une pensée interdisciplinaire voire transdisciplinaire comme celle de la pensée complexe (Morin, 2014).

\section{Pilier 3 : Prise en compte des particularités territoriales}

13 Le TA construit ne doit pas être l'héritier de la forme scolaire, "indifférente aux différences » (Garnier, 2020, p. 44), mettant à l'écart l'École des particularités et besoins territoriaux, mais doit au contraire s'y ancrer et s'y développer. Si pour l'École française, « élever, c'est sortir de sa condition, s'extraire de son milieu pour devenir soi, membre d'une communauté humaine plus large [...]» (Garnier, 2017, p. 25), autrement dit si l'ancrage territorial est indésirable, nous émettons l'hypothèse qu'au contraire ce dernier est le substrat même de l'émancipation. Nous considérons l'émancipation comme synonyme de l'empowerment ou « capacité d'affranchissement, de mobilisation, d'initiative et de contrôle, par appropriation ou (ré)appropriation d'un pouvoir sur la réalité quotidienne» (Maury et Hedjerassi, 2020, p.3). Cette hypothèse ne sera complètement vérifiée que dans la seconde partie de la recherche étudiant les impacts de l'action sur les profils d'écocitoyenneté des élèves, non détaillés dans l'article. 
14 En effet, pour Bruno Garnier, le territoire est un facteur omniprésent, agissant sur les individus et façonnant les éléments fondateurs de leur identité : «Avant que l'École puisse former, en l'être individuel, un être social, le territoire d'origine ou le territoire de résidence de l'individu a déjà forgé des références culturelles qui structurent son identité. » (Garnier, 2017, p. 25). À ce titre, nous considérons qu'une École développant l'ancrage territorial (social comme patrimonial) des élèves, permettrait à ces derniers de mieux connaître leur territoire, donc de mieux dessiner les traits de leurs identités individuelle et collective. En effet, nous considérons que pour s'émanciper, soit «sortir de sa condition, s'extraire de son milieu ", « devenir soi, membre d'une communauté ", encore faut-il connaître les caractéristiques de ce milieu et de cette communauté, autrement dit les caractéristiques de ce territoire. Ce n'est qu'une fois outillés de ses propres schémas identitaires qu'il est possible de s'émanciper. L'ancrage territorial ne serait donc pas un danger mais la première étape du processus d'émancipation.

Par ailleurs, pour François Flahault (2006), une évolution de paradigme pédagogique est nécessaire. Ce dernier critique le traditionnel triangle pédagogique (Houssaye, 2015), insuffisant pour donner sens aux apprentissages. Il propose de le transformer en un « carré pédagogique » via l'ajout d'une quatrième dimension, l'environnement, réalité concrète de vie. Le TA modélisé intègre la dimension environnementale proche ou territoriale et la met à l'épreuve. Ainsi, le territoire, au-delà de constituer un contexte et un outil éducatif, devient un " acteur éducatif » (Barthes et Champollion, 2012, p. 84) au même titre que l'enseignant, et le quatrième pôle d'un carré pédagogique territorialisé (sujet, objet, agent éducatif, territoire). Plus précisément, le TA se situe dans un courant d'éducation relative à l'environnement appelé éco-ontogénèse, s'intéressant «à la genèse d'un être humain dans ses relations avec l'environnement et plus particulièrement, des incidences de ces relations à l'environnement dans son développement, dans son ontogenèse » (Berryman, 2003, p. 3). Le territoire, considéré comme milieu de vie (Barthes et Blanc-Maximin, 2017), aux dimensions à la fois matérielle et symbolique, influence la genèse de l'être, de sa naissance à sa mort. L'objet de la recherche consiste à étudier un volet de l'ontogenèse, les liens patrimoniaux, en fonction des liens tissés avec l'environnement local ou territoire.

\section{Le modèle opérationnel de la stratégie éducative proposée : mise en place d'un territoire apprenant articulé à la forme scolaire}

16 Le schéma suivant (figure 1) compare la forme scolaire traditionnelle à gauche, et la stratégie éducative proposée à droite : 
Figure 1 : Synthèse comparative de la forme scolaire et de la stratégie éducative proposée.

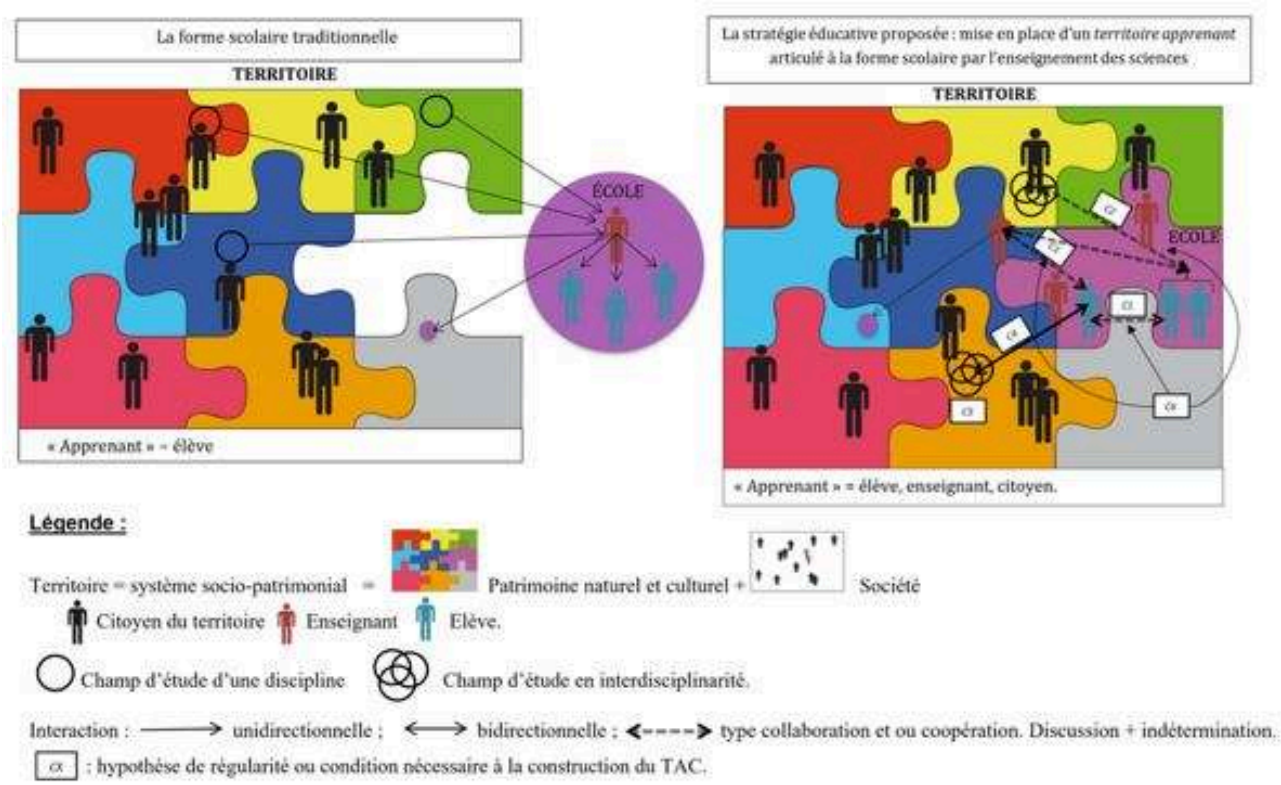

Dans la forme scolaire traditionnelle, illustrée par le schéma de gauche, l'École est représentée dans une bulle, entité distante du territoire. Les liens École-territoire sont majoritairement unidirectionnels et s'effectuent par l'intermédiaire de l'enseignant. Le professeur de sciences peut ponctionner certains éléments du territoire pour illustrer ses séances, visant ainsi un enseignement plus motivant. La transmission est unidirectionnelle, de l'enseignant vers les élèves. Les interactions directes inter-élèves et élèves-territoire sont respectivement restreintes et anecdotiques. Les élèves sont déconnectés de leur territoire (de leur patrimoine et de leur société). À quelques occasions, l'École peut être transférée sur le territoire comme lors de sortie scolaire. Enfin, pour certaines occasions non représentées sur le schéma, des intervenants horsÉcole peuvent interagir avec les élèves, comme les agents de la réserve citoyenne.

Dans la stratégie éducative proposée, schématisée à droite, un TA est initié à partir d'un établissement et articulé à la forme scolaire par l'enseignement des sciences. Nous avons souhaité faire évoluer l'École vers un mode plus ouvert et intégré dans son territoire, mais également fonder cette intégration sur un mode de socialisation démocratique. Pour cela, le TA repose sur six conditions ou hypothèses de régularité. La première condition ou « $\mathrm{C} 1$ », vise le renforcement et le développement de liens interélèves. $\mathrm{La}$ " $\mathrm{C} 2$ », le développement, maintien et utilisation de nouveaux liens élèvescitoyens. $\mathrm{La}$ " $\mathrm{C} 3$ », le développement, le maintien et l'utilisation de nouveaux liens élèves-enseignants. $\mathrm{La}$ « $\mathrm{C} 4$ » développe les interactions élèves-patrimoine. $\mathrm{La}$ 《 $\mathrm{C} 5$ » impose le fonctionnement en interdisciplinarité et la « $\mathrm{C} 6$ » exige des liens de nature coopérative et collaborative, ouvrant la voie à un nouveau mode de socialisation.

Plus précisément, une équipe d'élèves choisit un thème d'étude du patrimoine local et le propose aux membres de l'organisation apprenante. Selon une démarche d'investigation rigoureuse, ils co-construisent une ressource de type texte ou vidéo sur le thème proposé avec l'aide d'autres acteurs : enseignants et experts locaux hors-École contactés. Le fonctionnement en TA impose également le partage des savoirs aux membres de l'organisation et au-delà, aux acteurs du territoire non impliqués dans le projet. 
20 La mise en œuvre du TA a donc nécessité la création d'un support de communication, faisant le lien entre les élèves et les citoyens du territoire. Celui-ci prend la forme d'une application mobile destinée au partage des ressources co-produites et aux échanges entre utilisateurs. Le projet initial prévoyait le partage des ressources sur un site internet. Les élèves ont cependant jugé ce mode de communication dépassé et ont proposé le partage de leurs productions sur une application mobile. La pertinence de leurs arguments était convaincante. Nous avons donc construit une application mobile respectant trois mesures de précaution. La première exigeait une application gratuite et disponible sur les principaux systèmes d'exploitation des smartphones. La seconde imposait un cadre éthique respectant les principes de laïcité et de neutralité commerciale. L'application devait donc exclure les encarts commerciaux ou prosélytes ainsi que les pop-ups publicitaires. La troisième mesure exigeait la sécurisation des échanges entre les utilisateurs. L'application est finalement née d'une collaboration avec une entreprise locale, Good Barber, permettant la création d'applications qui respectent ces mesures.

21 Nous considérons que la mise en œuvre des six conditions précitées permet de mener une action éducative répondant aux exigences de territorialisation éducative, de coéducation et de socialisation démocratique. Les liens élèves-enseignants-citoyens sont bidirectionnels, modifiant les rapports de chacun aux savoirs. Dans le TA, des liens École-territoire directs sont tissés et d'autres indirects en découlent, par exemple via l'association $\mathrm{C} 2+\mathrm{C} 4$, association des liens élèves-citoyens hors-École et élèvespatrimoine. Ainsi, l'École (ses élèves) pourrait bénéficier du territoire, mais ce dernier, ses composantes sociale et patrimoniale pourraient également bénéficier de l'École. Ce $T A$ serait-il à l'origine d'une symbiose, entendue comme une association à bénéfice réciproque entre l'École (les élèves qui en sont les constructeurs) et le territoire (élèves et adultes qui en bénéficieraient) ?

La démarche choisie, ou "manière de conduire une action, de progresser vers un but " (Develay et Meirieu,1994, p. 161-162), est celle de l'intelligence territoriale (IT). Celle-ci peut être considérée comme une démarche de développement ascendante, initiant une synergie entre les acteurs locaux via l'identification d'une problématique locale commune et visant l'émergence d'une forme d'intelligence collective dans le cadre de projets de développement territoriaux (Bertacchini, 2004; Bertacchini, Girardot et Gramaccia, 2006 ; Pelissier et Pybourdin, 2009 ; Angelini, 2010). Le TA et l'IT présentent donc la même finalité - valoriser le développement territorial -, les mêmes ressources, soit les compétences des acteurs locaux, et le même principe fondateur, celui de la synergie des acteurs autour d'une problématique territoriale commune, aboutissant à l'émergence d'une intelligence collective. Dans ce cas, nous précisons que les élèves appartenant à la composante sociale du territoire, l'expression «développement territorial " intègre leur développement éducatif. Par conséquent, l'expression développement territorial n'est pas synonyme d'une conception utilitariste de l'éducation. Ainsi, nous considérons l'IT comme une démarche pertinente, une " aide à l'action " (Girardot, 2004, p. 2), offrant des principes de réflexion pour la conception d'un TA dont les premières finalités sont éducatives. Nous avons choisi d'assurer la conception et la mise en œuvre du $T A$ en exploitant les travaux cadrant les principes éthiques et méthodologiques nécessaires à la démarche d'IT (Girardot, 2004; Dessagne, Piot et Robin, 2019). Nous avons également fait appel aux travaux relatifs à la construction 
d'organisations apprenantes (Senge, 2015; Scharmer, 2016), qui nous ont permis d'établir un protocole spécifiquement adapté à la mise en œuvre de notre TA.

\section{La stratégie éducative proposée versus la forme scolaire}

Une analyse de la stratégie éducative proposée au regard de la forme scolaire nous permet de faire les constats suivants.

\section{Entre filiation et rupture avec l'éducation au développement durable}

La stratégie éducative proposée converge sous de nombreux points avec les " éducations », et plus précisément l'éducation au développement durable (EDD). Elles partagent l'objectif de transformation sociétale ainsi que de grands principes d'action (Dussaux, 2011). En ce sens, nous considérons qu'il existe une filiation entre l'EDD et la stratégie modélisée. L'EDD, qui va à l'encontre des caractéristiques de la forme scolaire sous divers angles d'attaque (comme la territorialisation et la coéducation), constitue la forme éducative préliminaire, posant les fondations des grandes orientations retrouvées dans notre stratégie de TA. Ce dernier constitue la phase évolutive postérieure, à la fois héritière de ces orientations et présentant des caractéristiques novatrices. En effet, il s'écarte de l'EDD sur deux points : la méthode d'application et la conception du partenariat. Concernant la méthode d'application, nous considérons que le $T A$ offre une nouveauté : un cadrage global et un plan d'action précis permettant aux projets éducatifs de réaliser leur plein potentiel. Pour Maryvonne Dussaux (2011), il s'agit d'ailleurs là de deux lacunes de l'EDD limitant ses impacts, amenant l'auteure à envisager les potentialités d'une stratégie de TA. Concernant la conception du partenariat, le TA s'inscrit également en rupture avec l'EDD. Les textes officiels de l'EDD de ces dernières années montrent une conception partenariale utilitariste (Bruxelle 2007, p. 4), le partenariat n'apportant pas nécessairement de bénéfice à chacun des acteurs. En revanche, le partenariat envisagé dans le TA est différent. Il s'agit d'un «partenariat apprenant» (Bruxelle 2007, p.2), que l'on pourrait qualifier de symbiotique. En effet, dans le cadre de la stratégie éducative que nous proposons, le savoir étant partagé et offert en ligne, chaque acteur du territoire, impliqué ou non dans le $T A$, peut y avoir accès et s'enrichir.

\section{La distance École-territoire et donc élèves-territoire}

Nous considérons que le TA s'attaque à cette distance via plusieurs voies. Il s'oppose dans un premier temps au caractère autonome de la relation sociale maître-élève et à sa conséquence directe, une vision de l'éducation scolaro-centrée. Diverses stratégies développant la coéducation ont permis d'estomper en partie la frontière Écoleterritoire et d'évoluer vers une vision moins scolaro-centrée. Le TA n'invente pas le principe de la coéducation sur lequel il est fondé, en revanche, il dépasse les stratégies précédentes en développant des coéducations inter et intragénérationnelles bidirectionnelles dans et hors de l'École. Il bouleverse alors les statuts des élèves et des adultes, abolissant la dualité sachant/apprenant, chacun pouvant être à la fois sachant et apprenant. Il tente ainsi de tendre vers une " société éducative » définie comme une société dans laquelle

[...] l'École n'est pas le seul lieu de l'éducation et [...] l'enseignant n'en est pas le seul responsable. C'est toute la société qui évolue, qui se construit, qui concourt au 
développement intégral de ses membres pour un développement social optimal.

(Sauvé 2001, p. 22)

élève. En effet, son fonctionnement exige l'établissement de liens maître-élève collaboratifs et coopératifs, impliquant la création de relations de confiance et de proximité. Ces liens sont étendus aux acteurs hors-École et nous considérons donc que le TA permet une réduction de la distance entre les élèves et la composante sociale du territoire. De plus, en produisant et partageant des ressources sur le patrimoine local, il réduit également la distance des élèves à la composante patrimoniale de leur territoire. Enfin, le $T A$ altère le fonctionnement traditionnel de la forme scolaire dans laquelle les thèmes d'étude sont dictés par des programmes scolaires nationaux. En effet, au sein du TA, les sujets d'étude sont non seulement territorialisés, mais également partagés de manière publique sur le territoire local. Ainsi, si la forme scolaire est largement dominée par l'éducation formelle, éducation « décontextualisée » (Rogers, 2007, p. 260) [traduction libre], nous considérons que le $T A$ force son évolution en y augmentant la part des éducations non formelles et informelles, respectivement «ajustées au contexte » et « contextualisées » (Rogers, 2007, p. 260) [traductions libres]. Il développe l'éducation non formelle par la territorialisation éducative, mais également l'éducation informelle par le partage de savoirs sur le territoire, chacun pouvant y accéder en fonction de ses besoins. Ainsi, nous considérons que le TA permet de réduire la distance École-territoire et donc élèves-territoire.

\section{Le mode de socialisation induit}

Le TA modifie les rapports au savoir : le savoir est offert dans et hors de l'École, est en lien avec le territoire; il est caractérisé par l'absence d'évaluation sommative et par une reconnexion du savoir à ses auteurs, autant de critères incompatibles avec la forme scolaire, la forçant à évoluer. Outre l'utilisation de nouvelles formes d'éducation, le TA construit permettrait la légitimation d'une nouvelle forme d'argumentation. En effet, dans la forme scolaire, les savoirs correspondent à une série d'affirmations à considérer par les élèves comme des vérités, qu'elles aient été démontrées ou non. Le savoir est alors qualifié de savoir "propositionnel», «[...] sous forme de propositions non logiquement connectées et qui se contentent d'énoncer des contenus." (Jorion et Dalbos, 2019, p. 5). De manière générale, privés de leur réflexe d'esprit critique, les élèves doivent donc accepter comme vraies les affirmations du maître car ce dernier représente l'autorité légitime à la transmission des savoirs. Selon Bernard Rey, cette privation de l'esprit critique induit deux dérives. La première consiste à légitimer l'argument d'autorité auprès des élèves, qui considèrent alors comme vrai tout ce qui vient de l'autorité. La seconde résulte de l'automatisation de la première, plongeant les élèves dans une "attitude de soumission intellectuelle» (Rey, dans Fillion, 2012, p. 173). L'enseignement des sciences, basé sur la démarche d'investigation, apparaît ainsi comme une parade au caractère accessoire de la démonstration rationnelle. Le TA, greffé à l'enseignement scientifique s'oppose également à ces dérives. En tant qu'auteurs des savoirs partagés, les élèves doivent être capables de réfléchir avec rigueur, raison, ouverture et esprit critique. Par conséquent, si, dans la forme scolaire, le savoir dominant légitimé est un savoir propositionnel, celui-ci n'est plus pertinent dans le TA. Ce dernier exige un savoir étoffé d'arguments rationnels, homologue au savoir « scientifique ». Il fait donc évoluer la forme scolaire d'une pratique régulière de

Éducation relative à l'environnement, Volume 16-2 | 2021 
l'argument d'autorité vers celle de l'argument rationnel. Ainsi, en stimulant chez les élèves leurs capacités d'échanges et l'usage de la raison, nous considérons que le $T A$ prépare davantage les élèves à la discussion (Vincent, 2008), et qu'il favorise donc un mode de socialisation démocratique.

Les finalités et les modalités de l'action exposées, l'objectif de la section suivante est de présenter la méthodologie de recherche associée.

\section{Méthodologie de la recherche}

Le volet recherche de cette recherche-action (RA) consiste en l'évaluation des impacts de la stratégie éducative proposée sur les liens patrimoniaux des élèves. Cette section situe d'abord l'étude dans le territoire de la Corse, puis explicite l'objectif, la démarche et les méthodes employées pour la récolte, le traitement et l'analyse des données.

\section{Territoire et population d'étude}

Le territoire étudié concerne la Corse, île française au cœur de la Méditerranée. Outre les particularités dues à l'insularité, les réalités géographiques offrent au territoire une diversité de paysages, avec un patrimoine naturel riche, mais on y observe également de profondes problématiques environnementales. Concernant le patrimoine culturel, la géographie a également permis de créer une diversité de micro-territoires de vie, à l'origine d'un patrimoine culturel riche et varié.

Les élèves interrogés sont des collégiens de cinquième et quatrième dont la moyenne d'âge se situe entre 13 et 14 ans. Au total, 128 élèves ont été interrogés, dont 64 ont constitué une cohorte test (TS), participant activement à la construction du TA, et 64 ont constitué une cohorte témoin (TM) n'y participant pas. Nous avons choisi de constituer les cohortes à partir de groupes-classe. En effet la RA menée a une «visée d'actionnabilité » (Allard-Poesi et Perret 2003, p.6): l'action doit pouvoir être réellement mise en œuvre et doit donc pouvoir s'articuler à la forme scolaire existante sans nécessiter son abolition complète. Le fonctionnement par groupe-classe y étant la règle, nous avons choisi de constituer les cohortes à partir de ces derniers. L'homogénéité entre les cohortes test et témoin est assurée par le mode de constitution des classes dont les règles exigent l'homogénéité interclasses. L'homogénéité intercohortes a été vérifiée par des analyses menées avant la mise en place de l'action : les résultats montrent des cohortes équivalentes à $\mathrm{T} 0$ et le recours à la statistique inférentielle confirme l'absence de différence significative.

\section{Objectif, démarche et choix méthodologiques}

L'objectif de recherche est d'évaluer les impacts de la stratégie éducative mise en œuvre sur les liens patrimoniaux des élèves. Pour cela, la démarche de recherche retenue est une analyse diachronique comparative, permettant la comparaison de l'évolution temporelle des liens patrimoniaux de cohortes d'élèves tests et témoins avant et après la mise en œuvre du TA. La recherche-action a donc été divisée trois temps :

- Le premier temps, ou temps T0, qui a principalement consisté en l'analyse des liens patrimoniaux avant la mise en œuvre de la stratégie éducative. 
- Le deuxième temps, ou temps $\mathrm{T} 1$, correspondant à la mise en œuvre de la stratégie éducative.

- Le troisième temps, ou temps $\mathrm{T} 2$, concentré sur la seconde analyse des liens patrimoniaux des élèves après l'expérimentation du TA.

Une fois la démarche identifiée, nous avons combiné deux méthodes d'étude complémentaires.

\section{Méthode d'étude 1 : Utilisation des représentations sociales patrimoniales pour l'analyse des liens patrimoniaux}

31 Afin de mettre au jour les liens patrimoniaux, nous avons choisi d'examiner les représentations sociales (RS) patrimoniales. En effet, une RS peut être définie comme « une façon de voir localement et momentanément partagée au sein d'une culture, qui permet de s'assurer l'appropriation cognitive d'un aspect du monde et de guider l'action à son propos » (Roquette dans Bloch et coll., 1997). Les RS reflétant la manière dont sont perçus et compris divers objets par la population étudiée, constituent des indicateurs de choix pour l'examen des liens patrimoniaux. Notre recherche consiste à établir puis à comparer les RS de la culture et de la nature corses des cohortes TS et TM à T0 et T2. Auront-elles des évolutions différentes? Autrement dit, la mise en place d'un TA aura-t-elle un impact sur leurs liens patrimoniaux? L'effet de la communication sur les RS a déjà été étudié par Mugny, Moliner et Flament en 1997. Ces chercheurs montrent qu'une communication infirmant un élément central, c'est-à-dire consensuel d'une RS, faite par un individu de haut statut, légitimant ses paroles, conduit à une évolution de celle-ci pour le groupe testé. Notre objectif est ici différent. Il s'agit de vérifier si une communication faite par des élèves, de statut non légitimé par la société actuelle pour une communication scientifique, pourrait influencer leur(s) RS, donc leur(s) lien(s) au patrimoine, voire influencer ceux des autres.

\section{Méthode d'étude 2 : Combinaison de différentes formes de triangulation}

Dans ses travaux, Thémis Apostolidis (2005) présente la triangulation théoricométhodologique comme une condition nécessaire à la validité des études sur les RS. Nous soutenons les propos de ce chercheur et présentons les formes de triangulation de notre étude dans le tableau 1 ci-dessous : 
Tableau 1 : Formes de triangulation de l'étude (Adapté d'Apostolidis, 2005)

\begin{tabular}{|c|c|c|}
\hline $\begin{array}{l}\text { Formes de } \\
\text { triangulation }\end{array}$ & Opérations de recherche & Objectifs \\
\hline $\begin{array}{l}\text { Triangulation du } \\
\text { chercheur }\end{array}$ & $\begin{array}{l}\text { - Sélection des techniques d'étude. } \\
\text { - Comparaison et discussion des règles } \\
\text { de traitement du corpus } \\
\text { (lemmatisation, encodage) en } \\
\text { collaboration avec un chercheur } \\
\text { qualifié en sciences de l'éducation et } \\
\text { du langage. }\end{array}$ & $\begin{array}{l}\text { Limitation du biais de } \\
\text { subjectivité du chercheur } \\
\text { par l'apport de regards } \\
\text { pluriels. } \\
\text { Optimisation de la validité } \\
\text { interne de l'étude. }\end{array}$ \\
\hline $\begin{array}{c}\text { Triangulation } \\
\text { méthodologique } \\
\text { d'ensemble }\end{array}$ & $\begin{array}{l}\text { - Méthode } 1 \text { : utilisation des RS du } \\
\text { patrimoine local pour l'analyse des } \\
\text { liens patrimoniaux. } \\
\text { - Méthode } 2 \text { : combinaison de } \\
\text { triangulations théorique et technique, } \\
\text { et du chercheur (Apostolidis, 2005) }\end{array}$ & $\begin{array}{l}\text { Optimisation de la validité } \\
\text { interne de l'étude. }\end{array}$ \\
\hline $\begin{array}{l}\text { Triangulation } \\
\text { technique }\end{array}$ & $\begin{array}{l}\text { - Technique } 1 \text { : Analyse quantitative } \\
\text { globale (Moliner et Guimelli, 2015)* } \\
\text { - Technique } 2 \text { : Analyse lexico-métrique } \\
\text { (Moliner et Guimelli, 2015)* } \\
\text { - Technique } 3 \text { : Analyse mixte } \\
\text { prototypique (Abric, 2005)* }\end{array}$ & $\begin{array}{l}\text { Multiplication des } \\
\text { perspectives d'analyse des } \\
\text { données et comparaison. } \\
\text { Optimisation de la validité } \\
\text { interne de l'étude. } \\
\text { * Test statistique de } \\
\text { significativité : optimisation } \\
\text { de la validité externe de } \\
\text { l'étude. }\end{array}$ \\
\hline $\begin{array}{l}\text { Triangulation } \\
\text { theorique }\end{array}$ & $\begin{array}{l}\text { - Théorie } 1 \text { : théorie du noyau central } \\
\text { (Abric, 2005) } \\
\text { - Théorie } 2 \text { : théorie du noyau matrice } \\
\text { (Moliner et Martos, 2005) }\end{array}$ & $\begin{array}{l}\text { Réduction des risques liés } \\
\text { aux limites des théories. } \\
\text { Optimisation de la validité } \\
\text { interne de l'étude. }\end{array}$ \\
\hline
\end{tabular}

\section{Protocoles de récolte, de traitement et d'analyse des données}

\section{Protocole de récolte des données}

Afin de réaliser l'analyse diachronique comparative, nous avons choisi d'utiliser des questionnaires de type panel (Durand et Blais 2016), permettant la comparaison de l'évolution temporelle de plusieurs cohortes. Les RS ont été recueillies en utilisant la "méthode de l'évocation hiérarchisée » (Abric, 2005, p. 63). Nous avons également prêté attention à la pression de normalisation à laquelle les sujets pouvaient être soumis, les forçant à cacher certains éléments de leur RS car contre normatifs. Cette pression, source de la «zone muette » ou «face cachée et non avouable» (Abric, 2005, p. 75) de la RS a été réduite par la "technique de la décontextualisation normative » (Abric, 2005, p. 78).

\section{Protocoles de traitement et d'analyse des données}

L'analyse quantitative globale ne nécessite pas de traitement particulier des données et consiste en une étude du nombre moyen de réponses par élève. L'objectif est donc de vérifier si les cohortes TS et TM ont un nombre de réponses moyen différent, autrement dit, si le TA influence quantitativement les liens patrimoniaux.

Concernant l'analyse lexico-métrique, le corpus est soumis à une correction orthographique ainsi qu'à un traitement de lemmatisation, aboutissant à l'élaboration d'une liste de mots ou d'expressions communs. Nous utilisons trois indicateurs, soit le nombre d'occurrence, le nombre de types et le nombre d'hapax, afin d'établir deux indices lexico-métriques descriptifs du corpus: l'indice de diversité et l'indice de 
variabilité (Moliner et Guimelli, 2015). Ces derniers n'ayant pas de valeur seuil, notre analyse porte sur leurs évolutions potentielles, de T0 à T2.

Pour l'analyse prototypique, le corpus est soumis à un traitement d'encodage pour lequel les mots porteurs du même sens ont été regroupés en catégories. Pour une analyse qualitative plus pertinente, les catégories sont regroupées en trois grands domaines d'expression selon la qualité du lien avec la nature ou la culture corse : les mots relatifs à la représentation d'un contenu, ceux qui relèvent d'une activité ou ceux qui témoignent d'un jugement personnel. Ici, la connaissance du contenu d'une RS est une étape nécessaire mais non suffisante à sa compréhension: il faut en effet considérer l'organisation du contenu.

Concernant l'étape de traitement des données, nous avons utilisé la théorie du noyau central (Abric, 2005), proposant l'utilisation combinée, pour chaque catégorie exprimée, de deux critères : la fréquence d'apparition et le degré d'importance accordé. La structure de la RS est obtenue selon les combinaisons du tableau 2 ci-dessous :

Tableau 2 : L'établissement de la structure d'une RS (Adapté d'Abric, 2005)

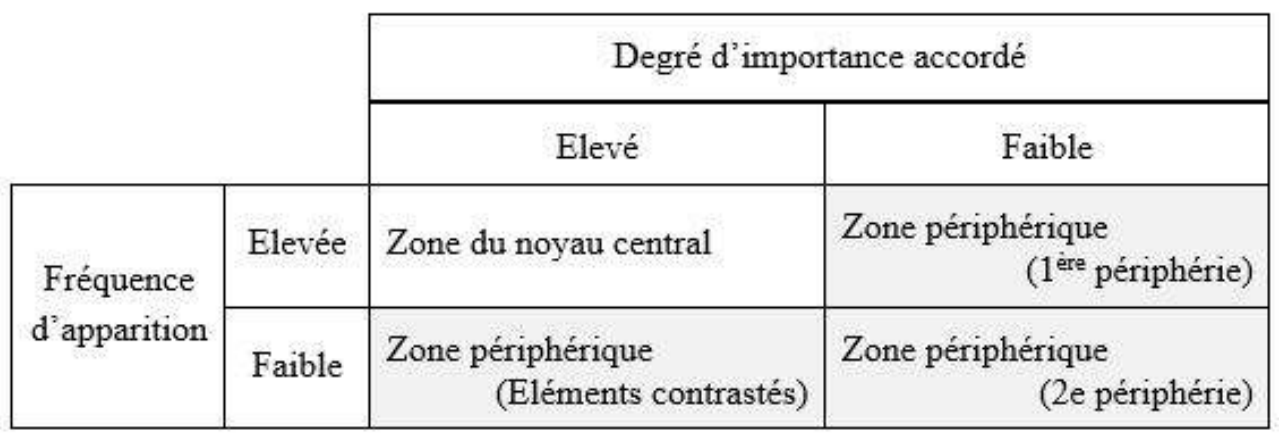

Concernant l'étape d'analyse, nous avons utilisé la théorie du noyau matrice de Pascal Moliner et Anaïs Martos (2005). Leurs travaux constituent une proposition d'amendement de la théorie du noyau central en ce qui concerne les fonctions attribuées aux éléments centraux et périphériques. Les éléments centraux seraient récepteurs de sens, jouant le rôle de matrice, intégrant les expériences individuelles et/ ou les contextes variés grâce à leur caractère polysémique, constituant un noyau consensuel de la RS. L'objectif de cette analyse est de comparer l'évolution temporelle des structures des RS des cohortes afin d'identifier l'impact potentiel de la stratégie éducative.

La méthodologie de recherche explicitée, nous présentons maintenant les résultats obtenus et les interprétations au regard de la problématique posée.

\section{Évaluation de la mise en œuvre de la stratégie éducative sur les liens au patrimoine}

Cette section synthétise et compare les résultats obtenus pour les trois techniques d'analyse. Elle propose ensuite une interprétation globale de ces derniers, identifiant les impacts de la stratégie éducative sur les liens patrimoniaux des élèves. 


\section{Résultats concernant les liens au patrimoine naturel corse}

Analyse quantitative globale: les cohortes semblent comparables à T0, alors qu'elles sont différentes à T2. La TS montre une évolution quantitative notablement différente. Afin de tester mathématiquement ces observations, nous avons soumis les résultats à la statistique inférentielle et avons effectué le test de Student pour deux échantillons indépendants. L'observation de cohortes TM et TS semblables à T0 est une condition nécessaire à la rigueur scientifique de l'étude : un écart TS/TM peut être constaté mais doit être statistiquement non significatif, c'est-à-dire uniquement dû à la variabilité intrinsèque de chaque cohorte. L'analyse statistique montrent des cohortes TM et TS sans différence statistiquement significative à T0 mais statistiquement différentes à T2 (au risque de $5 \%$ ). Les cohortes TS/TM ont une évolution temporelle différente, la TS ayant une évolution plus conséquente. L'analyse montre donc un impact quantitatif du TA sur les liens au patrimoine naturel de la TS.

41 Analyse lexico-métrique : les écarts des indices lexico-métriques semblent faibles à T0 et plus élevés à T2. Le test statistique du $\mathrm{Chi}^{2}$ montre des cohortes TM/TS sans différence statistiquement significative à T0 mais statistiquement différentes à T2 (au risque de $5 \%)$. Cette analyse confirme donc les résultats précédents en mettant en avant une évolution TS/TM différente. Elle permet également d'identifier un impact qualitatif du $T A$ sur les liens patrimoniaux.

Analyse mixte prototypique: concernant le volet quantitatif, les écarts TS/TM de volume total de la RS et du nombre de spécificités sont nuls ou faibles à T0 et augmentent à T2. La TS se distingue par l'apparition d'un plus grand nombre de catégories nouvelles. Cependant, les valeurs n'étant pas des proportions, il est impossible d'effectuer un test statistique et nos résultats ne concernent donc que l'échantillon présent, sans possibilité d'extension et de généralisation à une population théorique. L'analyse prototypique confirme les résultats des analyses précédentes et en renforce la robustesse : le $T A$ a un impact quantitatif positif sur les liens au patrimoine naturel des élèves TS ; leur RS sont davantage enrichies en nouveautés et spécificités. Concernant le volet qualitatif, la TS présente l'intégralité des nouveautés dans la zone périphérique. Parmi ces nouveautés, $54 \%$ sont corrélables aux éléments partagés par le TA et $36 \%$ sont relatives au domaine du jugement personnel. Pour la TM, l'intégralité des nouveautés apparaît également dans la zone périphérique, confirmant son rôle de "pare-chocs " (Moliner et Guimelli, 2015, p. 25) de la RS. En revanche, aucune des nouveautés n'est ni corrélable au TA, ni au jugement personnel. Le test exact de Fisher et l'analyse de la significativité par case montrent qu'à T0, les cohortes test et témoin présentent une distribution de leur RS en grands domaines statistiquement équivalente. À T2, seul la TS présente une distribution significativement supérieure pour le domaine du jugement personnel (au risque de $5 \%$ ). Nous mettons donc en évidence un impact qualitatif du TA sur les liens au patrimoine naturel des élèves TS se traduisant par l'apparition de catégories nouvelles relatives au jugement personnel. En conclusion, la stratégie éducative a permis d'enrichir quantitativement et qualitativement les RS et donc les liens au patrimoine naturel des élèves TS, actifs et constructeurs du TA. 


\section{Résultats concernant les liens au patrimoine culturel corse}

Analyses quantitative globale et lexico-métrique: les cohortes TS et TM semblent comparables et sans différence statistiquement significative à T0 comme à T2. Aucun impact du TA n'est donc identifié pour les liens au patrimoine culturel.

Analyse prototypique : concernant le volet quantitatif, les écarts TS/TM augmentent de T0 à T2 et la cohorte TS se démarque clairement par des évolutions quantitatives plus importantes. Comme pour la RS de la nature corse, le TA semble donc avoir un impact quantitatif sur la RS de la culture corse de la TS: RS enrichie en nouveautés et spécificités.

Concernant le volet qualitatif, nous constatons l'apparition de nouveautés corrélables au TA et de nouveautés relatives au jugement personnel dans les cohortes TS et TM. Une observation concordante avec celle de la RS de la nature corse aurait montré l'apparition de ces catégories uniquement dans la TS, démontrant ainsi l'impact de la construction active du TA sur les élèves de la TS. Dans le cas de la culture corse, la construction active d'un TA n'est donc pas le facteur à l'origine du développement des catégories relatives au jugement personnel. Leur apparition dans les deux cohortes estelle simplement due à "l'évolution normale» des enfants, qui, avec le temps, développent progressivement des capacités de jugement et d'affirmation de ce dernier? La proposition d'interprétation pour la RS de la nature corse n'est donc pas confirmée par celle de la culture corse, mais au-delà, les observations semblent antagonistes, ce qui nous conduit à leur réexamen.

Nous avons donc mené une investigation plus poussée et avons croisé ces données selon la participation au TA, distingué selon trois degrés permettant d'identifier trois nouvelles cohortes : la TS, où les élèves sont actifs et constructeurs du TA; la TMP, sous-groupe de la cohorte TM dont les élèves sont passifs, "profiteurs » du TA (consultation de l'application mobile de manière autonome et externe à l'établissement) et la TMN, sous-groupe de la TM dont les élèves ont une participation nulle (aucune consultation de l'application mobile).

47 L'examen des RS pour chacune de ces cohortes amène aux observations suivantes : à T2, la TMP présente des nouveautés corrélables au TA, dont certaines sont relatives au domaine du jugement personnel. À l'opposé, pour la TMN, aucune des nouveautés n'est ni corrélable au TA ni relative au jugement personnel. Eclairés par le degré de participation au $T A$, les résultats obtenus pour les RS de la nature et de la culture corses deviennent compatibles. Les résultats de la RS de la culture corse ne conduisent pas à rejeter les interprétations élaborées pour celles de la nature corse mais à les affiner pour proposer une interprétation plus complexe de l'impact du TA. Ce dernier est en réalité identifiable de manière différentielle selon les degrés de participation. Pour la TS, dans lequel les élèves sont actifs et co-constructeurs du TA, l'apparition de catégories nouvelles est plus élevée que dans la TM. Les nouveautés sont relatives aux contenus, aux activités, mais également au jugement démontrant ainsi l'évolution des élèves vers la prise de positions personnelles et l'affirmation de ces dernières. Ces nouveautés peuvent d'ailleurs apparaitre au niveau du noyau central reflétant un changement profond de la RS du groupe. A contrario, la TM présente moins de nouveautés. Les catégories relatives au jugement sont soit absentes, soit présentes dans la zone périphérique, démontrant une évolution moins profonde que celle opérée pour la TS. Leur apparition dans la TM ne concerne que les élèves ayant profité passivement 
du TA. Le TA peut donc également avoir un impact sur les élèves "passifs ", qui consultent l'application en autonomie. Nous mettons donc en évidence l'apprentissage de la cohorte TMP selon un mode informel, selon l'initiative des élèves, répondant à un réel désir d'apprentissage. Le TA a donc fonctionné puisqu'il a bénéficié à des acteurs du territoire non impliqués dans le partenariat.

\section{Conclusion}

La stratégie éducative modélisée et mise en œuvre, basée sur la construction d'un territoire apprenant initié depuis un établissement scolaire, se situe plus spécifiquement dans l'un des courants de l'éducation à l'environnement, celui de l'éco-ontogenèse. Celui-ci considère que le développement des élèves, en l'occurrence leurs liens patrimoniaux peuvent être influencés par les liens tissés avec l'environnement proche ou territoire. Les résultats de cette recherche mettent en avant un impact de la stratégie éducative sur les liens patrimoniaux des élèves actifs, constructeurs du territoire apprenant. Les représentations sociales patrimoniales de ces derniers sont quantitativement enrichies et qualitativement modifiées, notamment avec l'apparition et la multiplication de catégories relatives au jugement personnel. Ces modifications sont également observées chez les élèves passifs, ayant consulté en autonomie les éléments partagés par le territoire apprenant. Ainsi, le territoire apprenant a permis la création d'un réseau d'information territorial bénéfique pour les élèves constructeurs comme pour les élèves passifs, non impliqués dans le projet. L'apparition et la multiplication de catégories relatives au jugement personnel sont interprétées comme un indicateur d'une plus grande ouverture des élèves à la discussion.

Ces premiers résultats de recherche nous engagent donc sur une nouvelle interrogation : in fine, une éducation à l'environnement envisagée dans la perspective d'un territoire apprenant peut-elle être à l'origine d'un profil d'écocitoyenneté plus engagé dans la discussion? Autrement dit, plus engagé dans un mode de réflexion rationnel et ouvert, empowered ou " en pouvoir d'agir » (Maury et Hedjerassi 2020)?

\section{BIBLIOGRAPHIE}

Abric, J-C. (2005). La recherche du noyau central et de la zone muette des représentations sociales. Dans J-C. Abric (dir.). Méthodes d'étude des représentations sociales (p. 59-80). Toulouse : Editions ÉRES

Allard-Poesi, F. et Perret, V. (2003). La Recherche-Action. Dans Y. Giordano (dir). Conduire un projet de recherche, une perspective qualitative (p. 85-132). Caen : Editions EMS

Angelini, J. (2010). De la complexité de l'intégration des acteurs dans le développement local : approche par l'intelligence territoriale. Le cas du projet d'extension de Bastia. Thèse de doctorat. Université Pascal Paoli à Corte, France. 
Apostolidis, T. (2005). Représentations sociales et triangulation : enjeux théoricométhodologiques. Dans J-C. Abric (dir.). Méthodes d'étude des représentations sociales (p. 13-35). Toulouse : Éditions ÉRES

Barthes, A. et Champollion, P. (2012). Éducation au développement durable et territoires : Évolution des problématiques, modification des logiques éducatives et spécificité des contextes ruraux. Éducation relative à l'environnement : Regards - Recherches - Réflexions, volume 10. Consulté sur https://journals.openedition.org/ere/1049

Barthes, A., Blanc-Maximin, S., Alpe, Y. et Floro, M. (17-19/11/2014). L'éducation au patrimoine : pourvoyeuse de savoirs et/ou au service des territoires? Colloque international à Rouen. Les « éducations à, un levier de transformation du système éducatif » Consulté sur https:// halshs.archives-ouvertes.fr/halshs-01183403

Barthes, A., Champollion, P. et Alpe, Y. (2017). Permanences et évolutions des relations complexes entre éducations et territoires. Londres : Éditions ISTE

Barthes. A. (2020). Territorialisation de l'éducation : quelles significations politiques. Dans P. Champollion (dir.). Territorialisation de l'éducation Tendance ou nécessité (p.1-6). Londres : Éditions ISTE

Barthes, A. et Blanc-Maximin, S. (2017). Quelles évolutions de l'école française face à l'éducation au patrimoine. Revue des sciences de l'éducation 43(1). Consulté sur https://www.erudit.org/fr/ revues/rse/1900-v1-n1-rse03267/1042075ar/

Barthes, A., Blanc-Maximin, S. et Dorier, E. (2019). Quelles balises curriculaires en éducation à la prospective territoriale durable ? Valeurs d'émancipation et finalités d'implications politiques des jeunes dans les études de cas en géographie. Éducation et socialisation, Les cahiers du CERFEE (51). Consulté sur https://journals.openedition.org/edso/5755

Becchetti-Bizot, C. (2018). L'expérience du territoire. Apprendre dans une société durable. Diversité, (191), 159-163.

Becchetti-Bizot, C., Houzel, G. et Taddei, F. (2017). Vers une société apprenante : Rapport sur la recherche et développement de l'éducation tout au long de la vie. Rapport à l'attention de Madame la ministre de l'Éducation nationale, de l'Enseignement supérieur et de la recherche. CRI de Paris.

Berryman, T. (2003). L'éco-ontogénèse : les relations à l'environnement dans le développement humain. D'autres rapports au monde pour d'autres développements. Éducation relative à l'environnement : Regards - Recherches - Réflexions, volume 4. Consulté sur https:// journals.openedition.org/ere/5129

Bertacchini, Y. (2004). Le territoire, une entreprise d'intelligence collective à organiser vers la formation du capital formel local. Communication et Organisation, (25),1-16.

Bertacchini, Y., Girardot, J-J. et Gramaccia G. (2006). De l'intelligence territoriale. Théorie, Posture, Hypothèses, Définitions. Ve colloque "TIC et Territoire : quels développements ? Université de Franche Comté, Besançon, France.

Bier, B. (2010). Territoire apprenant : les enjeux d'une définition. Spécificités 3 (1),7-18.

Bloch, H., Dépret, E., Gallo, A., Garnier, Ph., Gineste, M-D., Leconte, P., Le Ny, J.-F., Postel, J., Reuchlin, M., et Casalis, D. (1997). Dictionnaire fondamental de la psychologie. Paris : Éditions Larousse

Bruxelle, Y. (2007). Le partenariat, entre réticences et fascination : quels questionnements éthiques pour l'institution scolaire ? Éducation relative à l'environnement : Regards - Recherches Réflexions, volume 6. Consulté sur https://journals.openedition.org/ere/3962 
Champollion, P. (dir.)., ECP., et ESO-Caen. (2020). Territorialisation de l'éducation Tendance ou nécessité. Londres : Editions ISTE group, Série Éducation, 5

Chaize, J. (2015, 15 juillet). L'organisation apprenante [vidéo en ligne]. Consultée sur https:// www.youtube.com/watch ?v =K2YLM8hTtxQ

Dessagne, D., Piot, C. et Robin, V. (2019). L'intelligence territoriale comme élément facilitateur des projets éducatifs en réseau : modèles, démarche et outils supports. HAL Archives ouvertes. Consulté sur : www.hal.archives-ouvertes.fr/hal-02314189

Develay, M. (2007). Donner du sens à l'école. Issy-les-Moulineaux : Éditions ESF Éditeur, 6e édition Develay, M., et Meirieu, P. (1994). Émile, reviens vite, ils sont devenus fous. Paris : ESF Editeur Durand, C., et Blais, A. (2016). Le sondage. Dans B. Gauthier. Recherche sociale; De la problématique à la collecte des données, 6e édition revisée. (p.469-516). Québec : PUQ

Durkheim, E. (2014). L'évolution pédagogique en France. Paris 14 : PUF, $3^{\mathrm{e}}$ édition

Dussaux, M. (2011). L'éducation au développement durable, l'école et les territoires apprenants. Thèse de doctorat. Université Paris Descartes, Faculté des sciences humaines et sociales à Paris.

Fillion, L. (2012). Éduquer à la citoyenneté : Construire des compétences sociales et civiques. Laon : Éditions RPA, Canopé-CRDP de l'Aisne

Flahault, F. (2006). Instruction, éducation et transmission entre générations. Revue du MAUSS 28 (2), 295-304.

Forquin, J-C. (2008). Sociologie du curriculum. Rennes : PU de Rennes

Garnier, B. (2017). Quelle place accorder aux territoires dans les politiques publiques d'éducation ? Dans A. Barthes., Ph. Champollion et Y. Alpe (dir.). Permanences et évolutions des relations complexes entre éducations et territoires (p.25-37). Londres : Éditions ISTE Group.

Garnier, B. (2020). Les enjeux de la territorialisation des politiques nationales d'éducation, entre replis locaux et appartenance au village-monde. Dans Malet, R. et Garnier, B. (dir). Éducation, Mondialisation et Citoyenneté : Enjeux démocratiques et pratiques culturelles (p. 39-57). Berne: Éditions Peter Lang.

Garvin, D.A. (1993). Building a Learning Organization. Harvard Business Review. Récupéré le 22 mars 2020 de : www.hbr.org/1993/07/building-a-learning-organization

Girardot, J-J. (2004). Intelligence territoriale et participation. $3^{\mathrm{e}}$ colloque TIC \& Territoire. HAL Archives Ouvertes. Récupéré le 22 mars 2020 de : www.hal.archives-ouvertes.fr/halshs-00539954

Houssaye, J. (2000). Le triangle pédagogique - Théorie et pratiques de l'éducation scolaire. Berne : Editions Peter Lang

Jambes, J-P., (2011). Interview de Jean-Pierre Jambes [vidéo en ligne]. Récupéré de https:// www.dailymotion.com/video/xloma3

Jorion, P. et Dalbos, G. (2019). La transmission des savoirs. Paris : Éditions de la Maison des sciences de l'homme

Lapassade, G. (2002). Observation participante. Dans J. Barus-Michel, E. Enriquez, et A. Lévy (dir.). Vocabulaire de psychosociologie (p. 375-390). Toulouse : Éditions Érès.

Maury, Y. et Hedjerassi, N. (2020). Empowerment, pouvoir d'agir en éducation. À la croisée entre théorie(s), discours et pratique(s). Spirale - Revue de recherches en éducation, 66, 3-13.

Moliner, P., et Guimelli, C. (2015). Les représentations sociales. Grenoble : Éditions PUG 
Moliner, P., et Martos, A. (2005). La Fonction Génératrice de Sens Du Noyau Des Representations Sociales. Une Remise En Cause ? Papers on Social Représentations 14. Récupéré le 15 février 2019 de : www.researchgate.net/publication/237596148

Morin, E. (2014). Introduction à la pensée complexe. Paris : Éditions Points.

Pelissier, M., et Pybourdin, I. (2009). L'intelligence territoriale. Les Cahiers du numérique 5 (4), 93-109.

Rogers, A. (2007). Non-Formal Education : Flexible Schooling or Participatory Education? Hong Kong : Éditions Springer

Sauvé, L. (2001). Le partenariat en éducation relative à l'environnement : pertinence et défis. Éducation relative à l'environnement, Regards- Recherches- Réflexions 3. Consulté sur https:// journals.openedition.org/ere/6603

Scharmer, O. (2016). La théorie U, renouveler le leadership : Inventer collectivement de nouveaux futurs. Gap : Éditions Yves Michel

Sébastien, L. (2014). Le territoire, un système socio-patrimonial décrypté par le modèle de l'Acteur en 4 Dimensions. Nouvelles perspectives en sciences sociales 10 (1), 283-329.

Senge, P. (2015). La cinquième discipline : Levier des organisations apprenantes. Paris : Éditions Eyrolles, 1 ère édition

Verdoni, D. (2013). L'inventaire du patrimoine en Corse : de la mémoire au projet, bilan et perspectives $1 / 2$. Conférence Université de corse. Consulté sur https://www.youtube.com/watch ?v =OEs0A5gNxOE Vincent, G. (1980). L'école primaire française. Lyon : Éditions PUL

Vincent, G. (2008). La socialisation démocratique contre la forme scolaire. Éducation et francophonie 36 (2), 47-62.

Vincent, G., Lahire, B., et Thin, D. (1994). Sur l'histoire et la théorie de la forme scolaire. Dans G. Vincent (dir). L'éducation prisonnière de la forme scolaire? Scolarisation et socialisation dans les sociétés industrielles (p.11-48). Lyon : Éditions PUL

\section{NOTES}

1. Référence de la recherche doctorale: Moretti, L. (2021) Enseignement des sciences, interdisciplinarité, territoire apprenant: Quelles articulations? Quelles modalités ? Quels résultats? Thèse de doctorat. Université Pascal Paoli à Corte, France.

\section{RÉSUMÉS}

Cet article présente les fondements, la méthodologie et les premiers résultats d'une rechercheaction, visant la modélisation, la mise en œuvre et l'évaluation d'une stratégie éducative basée sur la construction d'un territoire apprenant (TA). Ce dernier est articulé à la forme scolaire par l'enseignement des sciences et initié depuis l'École. Cette recherche s'intéresse aux effets potentiels du TA sur les liens patrimoniaux des élèves. L'environnement proche - ou le territoire 
et plus précisément, sa composante patrimoniale locale - est considéré comme levier de développement éducatif et valorisé ici via une médiatisation numérique publique, notamment par la création d'une application mobile. Les résultats de la mise en œuvre de la stratégie éducative montrent des impacts positifs sur les liens patrimoniaux des élèves actifs, constructeurs du TA, mais également sur ceux des élèves passifs, non impliqués dans le projet.

This article presents the foundations, the methodology and the first results of an action research, aimed at the modelling, implementation and evaluation of an educational strategy based on the construction of a learning territory (TA). The latter is articulated to the school form by the teaching of sciences and initiated from the School. This research focuses on the potential effects of TA on students' patrimonial ties. The nearby environment, or territory, and more precisely its patrimonial component, is considered as a lever for educational development and valued here via public digital media coverage, in particular through the creation of a mobile application. The results of the implementation of the educational strategy show positive impacts on the patrimonial links of active students, builders of $T A$, but also on those of passive students, not involved in the project.

\section{INDEX}

Mots-clés : enseignement des sciences, territoire apprenant, éco-ontogenèse, liens patrimoniaux, représentations sociales

Keywords : science teaching, learning territory, eco-ontogenesis, patrimony links, social representations

\section{AUTEUR}

\section{LAURE MORETTI}

Laure Moretti est la fois doctorante au sein de l'Unité Mixte de Recherche « Lieux, Identités, eSpaces et Activités » (UMR-CNRS 6240 LISA) de l'Université de Corse et praticienne, enseignante de sciences dans le secondaire et le supérieur. 